\title{
¿ESSE 0 EXISTENCIA? \\ LA DISTINCIÓN REAL ENTRE LAWRENCE \\ DEWAN Y ÉTIENNE GILSON
}

\author{
Esse or Existence? \\ The Real Distinction between Lawrence \\ Dewan and Étienne Gilson
}

\author{
Manuel Alejandro Serra Pérez \\ Universidad de Murcia
}

\section{Resumen}

Uno de los temas más debatidos en el período inmediatamente posterior a la muerte de Tomás de Aquino fue cómo interpretar fielmente de acuerdo a sus textos el concepto de esse, dada la cualidad metafísica particular que éste adquiría en esta nueva ontología. Teniendo en cuenta que durante la escolástica los pensadores trataban de servirse de la filosofía como instrumento para explicar la fe, la comprensión del esse como distinto realmente de la esencia, fue el centro del debate por ser la base del principal argumento para mostrar la diferencia ontológica entre Dios y las criaturas.

Palabras clave

Esse; esencia; existencia; Dewan; Gilson

\begin{abstract}
One of the most debated issues in the period immediately following the death of Thomas Aquinas was how to interpret faithfully according to his texts the concept of esse, given the particular metaphysical quality that it acquired in this new ontology. Bearing in mind that the scholastic thinkers tried to use philosophy as an instrument to explain Christian faith, the understanding of esse as distinct from essence, was the center of the debate for being the reason of the main argument to state the ontological difference between God and creatures.
\end{abstract}




\section{Keywords}

Esse; essence; existence; Dewan; Gilson

\section{Introducción}

La teoría del acto y la potencia de Aristóteles parecía haber llegado a su máximo nivel de profundidad con la dialéctica «materia-forma», enraizada en la comprensión de la substancia como aquello que es gracias a la forma. En este paradigma metafísico que parte de la concepción de la eternidad del mundo y, por tanto, de la materia, la Causa primera, concebida como Acto puro de pensamiento, era sin duda responsable de la substancialidad de los entes, pero jamás pudo siquiera plantearse la cuestión de la existencialidad de éstos, entendida como paso ontológico cualitativamente crucial de la nada al ser, no al ser substancial, es decir, no al ser tal o cual cosa, sino a la existencia como tal. Esta temática no pudo de hecho suscitarse sino bajo un nuevo paradigma, el cristiano, donde la creación era el inicio de una revolución filosófica que iba a poner a todos los filósofos a estudiar en otra dirección. El inicio temporal de los seres y del mundo por la acción de Dios, identificado ahora con la noción de ser, puso en evidencia la fundamental distinción ontológica en los entes entre ser y esencia. Si bien desde el inicio la tradición patrística se planteó, especialmente con Boecio y el Pseudodionisio, esta distinción, fue el filósofo árabe Avicena el que con claridad evidenció la impor-tancia que había de darse a la existencia como un elemento distinto de la esencia. Pero, con quien esta problemática se hizo célebre y fue expuesta con más rigor y claridad fue con Tomás de Aquino, quien quiso sintetizar, a partir de las intuiciones de Avicena y dentro de un pensamiento cristiano, lo más válido de las doctrinas de Platón, Aristóteles y Plotino, llegando así a la concepción de la identidad en Dios de esencia y ser, la causalidad eficiente a través del esse y la participación metafísica de los entes en el Esse puro.

La muerte de santo Tomás dejó en manos de sus seguidores la delicada tarea de conservar con fidelidad el recto sentir de sus nociones más importantes y las fórmulas que las expresaban. Sin embargo, la corruptio notionum fue inevitable, y tanto el concepto de esse como la célebre fórmula de la distinctio realis se malogró en dos sentidos, $1^{\circ}$ por la reducción de esse a la mera existencia fáctica; $2^{\circ}$ consecuentemente, al hacer del esse una cosa (esencia), esta distinción no pudo defenderse como real sino a lo sumo de razón (Suárez). En este período la diferencia entre ser y esencia era incuestionable porque era el único camino que se veía para distinguir a Dios de las criaturas, algo que ningún pensador cristiano ponía en duda, pero la pérdida de su sentido metafísico correcto será una de las causas por las que, en tiempos recientes, Heidegger considerará la metafísica occidental (incluyendo injustamente a Tomás de Aquino) como ignorante del ser en detrimento del ente, algo que sólo podría pensarse leyendo a Tomás a través de muchos de sus comentadores, pero no a él mismo. 
Pues bien, el siglo XX, y precisamente gracias a esta crítica de Heidegger (en parte muy bien acogida y acertada), vio renacer la metafísica del ser tanto en Aristóteles como en santo Tomás. Con ellos, uno de los temas que ha vuelto a centrar la atención de algunos estudiosos ha sido el del ser respecto del ente, lo que, a fin de cuentas, termina siendo el planteamiento de la distinción real como modo de entender esta relación.

Dentro del mundo del tomismo contemporáneo, y al margen de la escuela analítica que ha tenido su relevancia, algunos autores han polemizado en torno a estas nociones y sus fórmulas. Esta es la razón por la que, en este artículo, quisiera dar voz a una confrontación que me ha parecido importante, y que tiene como autores a dos tomistas reconocidos, Lawrence Dewan y, sobre todo, el medievalista francés Étienne Gilson. A través de un artículo llamado Gilson and the Actus Essendi, el Canadiense quiso salir al paso de algunas de sus tesis defendidas en su libro Elementos de Filosofía Cristiana, planteando una serie de preguntas. ¿Defendió Tomás de Aquino una distinción real de esencia y ser en el ente? ¿Puede ésta ser demostrada filosóficamente? En caso afirmativo, ¿cómo podríamos articular un argumento adecuado para ello? Por otro lado, como ya hemos indicado, la polémica en torno a la identificación del esse tomista con la existencia real de la esencia ha sido otro de los temas más discutidos entre autores, y también está presente en esta confrontación, dada su vinculación con la demostrabilidad de la distinción real.

Para afrontar este cometido hemos dividido el artículo en dos partes bien diferenciadas. En la primera vamos a tratar de exponer los temas principales del debate con la respectiva argumentación de cada autor. Por nuestra parte, si bien iremos anotando críticamente la posición que nos parece más cercana a santo Tomás, dejaremos para una segunda parte una exposición más sistemática de los principales argumentos que se van desarrollando, apoyándonos en especialistas reconocidos tanto del siglo XX como actuales, que han dedicado su carrera al estudio del pensamiento del Aquinate. Finalmente presentaremos brevemente unas conclusiones.

\section{Esencia y esse en la confrontación entre Dewan y Gilson}

Dewan dedica la segunda parte de su artículo Gilson and the actus essendi ${ }^{1}$, que aborda algunas ideas importantes del tomismo de Étienne Gilson presentes en su libro Elementos de Filosofía Cristiana, a la discutida cuestión de la distinción real entre esencia y ser. En este primer punto de nuestro trabajo vamos a exponer, por un lado, los argumentos que el Canadiense desarrolla para mostrar que el modo de enfocar este tema por parte de Gilson es erróneo y, por otro, la confrontación de estas observaciones suyas con el tomismo del medievalista francés. Las ideas que expondremos son: a) demostrabilidad de la distinción real desde la substancia causada y, b) distinción y relación entre esse y existencia.

${ }^{1}$ Dewan, L., «Gilson and the Actus Essendi», Études Maritainiennes/Maritain Studies, 15 (1999), pp. 70-96. 


\section{a) Demostrabilidad de la distinción real desde la substancia causada}

El primer argumento que Lawrence Dewan se propone cuestionar de esta parte de Elementos de filosofía cristiana, comienza con el acercamiento a la naturaleza real del esse. A su modo de entender, Gilson, cuyo objetivo es siempre ir más allá de la esencia, nos dice que algunos filósofos han entendido que el esse de Tomás de Aquino no sería más que la esencia puesta en acto dando como resultado la cosa existente, en cuyo caso, lo que se conoce como actus essendi se identificaría con la existencia real de la substancia ${ }^{2}$. Pero, a lo que conduce esta cuestión es a lo que podríamos denominar el núcleo central de lo que Dewan quiere debatir, que es el tema de la demostrabilidad de la distinción real de esencia y ser. A lo primero, el Canadiense se muestra conforme con la conclusión de Gilson a la hora de establecer de un modo claro y directo que, en la doctrina de Tomás de Aquino, a tal cosa se llega sin dificultad: «to those who perceive that to be is, in every thing, the ultimate act that causes it to be a being, the demonstration becomes crystalclear. One should rather say that there is nothing left to demonstrate» ${ }^{3}$. Sin embargo, poco más tarde, el Francés afirma que el Aquinate, de la mano de Avicena, lo que viene a mostrar con la via remotionis es exclusivamente la posibilidad de conocer la existencia de Dios y la identidad en Él de esencia y ser, mas la distinción real en los seres de esencia y ser no puede ser demostrada a partir de la substancia:

The way of remotion therefore leads to the notion of a God Whose essence is his very act of existing (...) And this does not seem philosophically demonstrable from the notion of substance alone. It can be demonstrated that no essence is the cause of its own existence, from which it follows that whatever has an essence, and exists, must exist in virtue of an external cause; but no one has ever been able to demonstrate the conclusion that, in a caused substance, existence is a distinct element, other than essence, and its act ${ }^{4}$.

Dewan señala que Gilson, a pesar de que está dispuesto a conceder que se puede probar que la existencia de la substancia le viene de alguna causa externa, sin embargo, no le da aquí al esse su auténtico valor, especialmente en orden a probar la distinción real, independientemente de que podamos saber más o menos sobre él ${ }^{5}$. Aunque no podamos partir de una distinción como tal a partir de la mera substancia, una vez que advertimos su composición, cuando contemplamos esta substancia como causada, entonces podemos conocer la distinción de esencia y ser:

It need not, at first, be distinguished from essence. Both terms, «essence» and «act of being», seem to refer to principles or elements of a being. It is only when the thing is seen as caused, and thus as dependent on a higher and more noble essence, that one

${ }^{2}$ Gilson, É., Elements of Christian Philosophy, New York, Doubleday and Co, 1960, p. 119. Más adelante se detallará la posición de los autores más relevantes.

${ }^{3}$ Ibid., p. 127.

${ }^{4}$ Ibid., p. 128.

${ }^{5}$ Dewan, «Gilson», op. cit., p. 84. Sin embargo, como trataremos de mostrar, lo que podamos saber de la naturaleza del esse es la clave hermenéutica del conocimiento de la distinción. 
must conceive of a distinction, within the caused thing, between its essence and its act of being (...). It is true that if one does not recognize esse as a target of metaphysical attention right from the start, one will never propose the Thomistic doctrine. Still, that does not mean one must fully know the nature of esse right from the start ${ }^{6}$.

Tras observar que Gilson no da al esse la debida atención, nos explica cómo es posible captar su sentido. En efecto, Dewan dice que «al principio no es necesario distinguirlo de la esencia», sino que es percibiendo la substancia como causada como se puede probar la distinción real en ella de esencia y ser, concluyendo poco después que «es cierto que si uno no reconoce desde el principio el esse como objeto de atención metafísica nunca propondrá la doctrina tomista». La pregunta que Gilson le plantearía inmediatamente sería: pero, ¿acaso al esse del que parte Tomás no se accede a través de la Metafísica del Éxodo ${ }^{7}$, donde encuentra una de las fuentes principales por las que es posible conocer esta noción originaria de toda su metafísica? ${ }^{8}$ De hecho, cuando Dewan se apoya en la causalidad eficiente para pretender probar la distinción real, parece no estar cayendo en la cuenta de que, si esta causa no es el Acto puro de Ser, entonces es el Acto puro de Aristóteles, el cual, a pesar de hacer proceder la substancialidad de los entes de este Primer Motor, nunca distinguió esencia y existencia, al contrario, concebía la esencia como esse in actu, esto es, la cosa existente por virtud de la propia esencia, la cual, en su metafísica, es acto supremo ${ }^{9}$. En resumidas cuentas, para llegar a la distinción real a partir de la causalidad de la substancia, si la naturaleza de esta Causa eficiente no es el Esse puro, no hemos desplazado la actualidad de la esencia al esse $e^{10}$. Si, en cambio, esta Causa es el Esse puro, entonces la distinción se fundamentaría en Él como Acto puro de Ser y no en una indeterminada causalidad de la substancia. Lo importante es destacar que, entonces, la distinción se puede mostrar teniendo en el horizonte no cualquier conocimiento del esse sino un conocimiento propio de éste como acto ${ }^{11}$. Comentar a Tomás y querer mostrar la distinción real sin el esse, el cual se fundamenta en el Esse puro, sería quedarse en la Metafísica.

${ }^{6}$ Ibid., op. cit., p. 84.

${ }^{7}$ Cf. Gilson, É., Dios y la filosofía, Buenos Aires, Emecé, 1945, p. 70.

8 «''esse come actualitas absolute è la novità della rivelazione dell'Esodo $(3,14)$, ignota al pensiero classico, e suppone perciò la creazione». Fabro, C., Partecipazione e causalità, Opere Complete, vol. 19, C. Ferraro (ed.), Roma, EDIVI, $2010^{2}$, p. 161. Otro texto de esta misma obra que subraya la capitalidad de esta idea: «Il capovolgimento operato da san Tommaso ha il suo nucleo genetico attorno alla nozione biblica di Dio: Sum qui sum (Ex. 3, 14): una nozione che a sua volta mediante la convergenza da lui operata fra i contrastanti princìpi platonici e aristotelici attinge supremamente la sua fondazione teoretica», Ibid., p. 649.

${ }^{9}$ "L'esse come positività assoluta e atto di ogni forma è la novità della metafisica di san Tommaso, ignota ad Aristotele infatti riversa la verità dell'őv-ens non sull'esse, come atto di ogni atto,

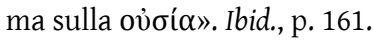

${ }^{10}$ Ibid., p. 41.

${ }^{11}$ «L'originalità dell'esse tomistico si profila chiaramente nella sua diretta derivazione biblica e nel plesso delle sue fonti dottrinali». Ibid., p. 179. 
En la edición revisada de su artículo, Dewan añade un texto en el que reelabora su argumentación. En primer lugar, afirma que el conocimiento inicial del esse a partir de la substancia es algo confuso, pero, por otro, dice lo contrario, que en sí mismo considerado no es difícil acceder a él. Después, al final del párrafo volverá a insistir en que a todos es bien conocido el ser del ente, pero que el problema está en querer saber qué es. En medio, para probar esta facilidad para conocer el esse, pone un ejemplo de cómo, a partir de un objeto sensible, conocemos el ser como viviente, reconociendo que se trata de una consideración general del esse. Y ya, cuando el intelecto pasa a una segunda fase donde comprende el ente en un sentido más profundo, llega así al primer principio básico del entendimiento, que es el de contradicción. Este es, pues, lo conocido por todos -concluye-. El problema vendría cuando queremos conocer qué es para desde ahí distinguirlo de cualquier otro elemento (en clara alusión con el método que está utilizando Gilson). Y afirma: «yo aplico lo que Tomás dice sobre el conocimiento del alma al conocimiento del esse».

Obviously, this knowledge of ens already includes a knowledge of esse, but that is still a somewhat confused knowledge of esse, since it is only as included in composite. However, knowledge of esse just in itself cannot be too difficult of access (...). That esse is, then, everyone knows. The problems arise when we seek to know what it is, i.e. to distinguish it from every other item with which it might be ${ }^{12}$.

Dewan dice que al principio tenemos un conocimiento confuso del esse que no nos permite conocer la distinción. Pero, cuando después recurre a la causalidad de la substancia para probar la distinción, no se da cuenta de cómo la noción de causalidad eficiente tomista presupone la noción de esse como acto, quedando así en entredicho su argumento ${ }^{13}$.

${ }^{12}$ Dewan, L., «Gilson and the Actus Essendi» (extended edition), International Journal of Philosophy, 1 (2002), pp. 65-99, en p. 82. A propósito de esta explicación, puede resultar oportuna la siguiente reflexión de Fabro: «Molti tomisti hanno ammesso e alcuni parlano espressamente di una certa intuitio abstractiva, una terminologia ignota a san Tommaso e di cui la stessa composizione (intuizione-astrazione) denuncia l'imbarazzo e il ripiego puramente verbale». Fabro, Partecipazione, op. cit., p. 65.

${ }^{13}$ Este argumento no es concluyente porque, ser causado implica que es a la luz de esa causa eficiente (que es Puro Ser) como deducimos la composición real de esencia y ser; luego, si la composición no se funda en ella misma en tanto que substancia ni en el mero hecho de ser causada, sino de ser causada por parte de una Causa eficiente que es Puro Ser, esta composición no se prueba -esto es, no se funda- ni en su ser substancia ni en el hecho mismo de ser causada, sino en la simplicidad de su Causa (Puro Ser), y, en tanto que simple (Puro), su efecto, la substancia, tiene que ser compuesta. Por tanto, sabemos que la substancia es compuesta no porque sea causada sino por la simplicidad de la naturaleza de su Causa. Luego lo que niega Gilson no es que no se pueda demostrar la composición, sino que esta demostración pueda realizarse por la propia substancia, y no por la vía remotionis, como hizo Tomás, esto es, por la demostración de la existencia de Dios y, en consecuencia, de la identidad en Él de esencia y ser. En definitiva, es la identidad del Acto Puro como ipsum Esse subsistens de donde parte el conocimiento de la naturaleza del ente, y, por tanto, de la distinción real. 
Dando un paso adelante, el Canadiense analiza más pormenorizadamente otro texto de Gilson a partir del cual va a debatir con él sobre la necesidad de comprender adecuadamente esta idea de la causalidad como medio para probar la distinción.

There is another feature of Gilson's above remarks which does not seem right. He says, concerning the real distinction (...). It is quite true that the real distinction between a substance or essence and its act of being cannot be demonstrated from the notion of substance alone (...). If one is speaking of efficient causality, it is of course true that nothing is the cause of itself; in that sense «no essence is the cause of its own existence». However, from that it does not follow that whatever has an essence and exists must exist by virtue of an external cause. The real distinction certainly does not follow from «no essence is the cause of its own existence». The truth is that to prove the real distinction, one begins with a thing's being caused (as by an efficient cause). The distinction can be proved from caused substance, but not from substance as substance or essence as essence ${ }^{14}$.

Dewan parte con Gilson del presupuesto de que, desde el punto de vista de la causa eficiente, nada es causa de sí mismo. Pero, inmediatamente introduce esta adversativa: «However, from that it does not follow that whatever has an essence and exists must exist by virtue of an external cause». Caigamos en la cuenta de que justo en el párrafo anterior había afirmado que «no essence is the cause of its own existence», lo cual resulta llamativo. Si ninguna esencia es la causa de su propia existencia es porque, si lo fuera, sería Dios (Ser por esencia); y, como ninguna substancia más que Él puede ser el Ser por esencia, de ahí se deriva que ninguna substancia es causa de su propia existencia; $\mathrm{y}$, si no es causa de su propia existencia, pero de hecho existe, su innegable existencia requiere necesariamente una causa externa. De hecho, el argumento de la cuarta vía, que Gilson desmarcó de la noción tomista de esse, prueba que, por la contingencia de los entes, llegamos a un Ser necesario que les da fundamento, pero de ningún modo que en ellos su existencia se distingue de su esencia ${ }^{15}$. En todas las vías se emplea la causalidad, que es lo

${ }^{14}$ Dewan, «Gilson», op. cit., p. 85.

${ }^{15}$ Cf. Gilson, É., «Elementos de una metafísica tomista del ser», P.J. Moya Obradors (trad.), Espíritu, 41 (1992), pp. 5-38, en p. 11. Ángel L. González es uno de los que, junto a Fabro y otros, han estudiado en profundidad la cuarta vía. Según estos autores, ésta se funda en el esse y trata de concluir en la existencia de Dios como Ser necesario por la contingencia de los entes. Al respecto, afirma González: «la primacía de la participación sobre la causalidad comporta la primacía -y fundación- del ser sobre la existencia. Si se entiende esto así, a mi juicio, -en cierto modo contra la opinión gilsoniana- no existe extrapolación de una prueba metafísica cuya andadura finaliza en Dios como Ser subsistente y Causa del ser». Y, en nota a pie añade citando a Gilson: «Después de demostrar [Tomás de Aquino] que existe un Dios, aún queda un largo camino antes de alcanzar esta suprema metafísica: un ser cuya misma esencia es ser». Y comenta de la afirmación gilsoniana: «de acuerdo en el último punto con el admirado maestro tomista; respecto de la opinión de que no se trata de una demostración de la existencia de Dios, véase nuestra opinión [remite a una parte de su estudio donde la confronta]». González, Á.L., Ser y participación, Pamplona, Eunsa, $2001^{3}$, p. 159. Personalmente me inclino hacia el escepticismo gilsoniano en este punto: el esse es un punto de partida y no el término de una demostración; de este modo, la prueba de la existencia 
que admite Gilson a partir de la sola substancia causada, pero, como hemos visto, de su causalidad no se deriva la distinción ${ }^{16}$.

¿Cómo se conjugan estas dos afirmaciones? Si desde la causalidad eficiente afirmamos que nada es causa de símismo, ¿cómo puede sostenerse que de aquí no se sigue que, aquello que tiene una esencia y existe, debe existir en virtud de una causa externa? ¿Acaso en ambas afirmaciones no se está diciendo lo mismo? Cuando afirmamos que «todo lo que tiene una esencia y existe debe existir en virtud de una causa externa», estamos corroborando lo que luego negamos, por cuanto que el hecho de poseer la esencia indica que su substancialidad tiene su origen no en sí misma sino en una causalidad externa a ella. En cualquier caso, el razonamiento de Gilson es el que parece haber utilizado santo Tomás:

It can be demonstrated that no essence is the cause of its own existence, from which it follows that whatever has an essence, and exists, must exist in virtue of an external cause; but no one has ever been able to demonstrate the conclusion that, in a caused substance, existence is a distinct element, other than essence, and its act ${ }^{17}$.

Pongamos ahora el foco de nuestra atención en esta conclusión de Dewan:

The real distinction certainly does not follow from «no essence is the cause of its own existence». The truth is that to prove the real distinction, one begins with a thing's being caused (as by an efficient cause). The distinction can be proved from caused substance, but not from substance as substance or essence as essence ${ }^{18}$.

La distinción puede probarse desde la substancia causada, pero no desde la substancia como substancia o esencia como esencia. Ahora bien, lo que él llama «substancia como substancia», ¿acaso no es lo mismo que la substancia como causada? La naturaleza de la substancia, en sí misma considerada, comporta necesariamente la causalidad, al menos de su substancialidad. La única Substancia de la que, como substancia, no deriva causalidad alguna, es la Incorruptible, el Motor inmóvil. Pero, como se viene insistiendo, de esta causalidad, que es la única que podemos conocer fuera del esse, no se deriva ninguna

de Dios, a la que se pretende llegar a través de la distinción real, a partir de la contingencia de los entes, plantea el mismo problema: la presuposición de la noción de esse, aplicada tanto al ipsum Esse como al esse creado. En cualquier caso, creo que es una cuestión aún abierta cuya definitividad (de la prueba) requiere de ulterior fundamentación.

${ }^{16}$ De algún modo, Gilson pretende circunscribir el objeto demostrativo de la cuarta vía a la existencia de Dios a través de la contingencia de los entes y su consecuente causalidad; pero de aquí no se deriva para él la distinción real. La distinción real, como expliqué anteriormente (nota 13), presupone la naturaleza de la Causa productora de los entes, y no la mera contingencia de éstos. Esta posición a la que se avino al final de su carrera el medievalista francés fue rechazada por casi todos los tomistas, incluso por los más admirados por él. Algunos ejemplos: Fabro, Raeymaecker, Forment y Á. L. González.

${ }^{17}$ Gilson, Elements, op. cit., p. 128.

${ }^{18}$ Dewan, «Gilson», op. cit., p. 85. 
distinción, y la causalidad transcendental del esse ya presupone y lleva consigo esta noción.

Esta conclusión a la que llega Gilson según la cual por la substancia causada no podemos probar que la existencia es un elemento distinto de la esencia y su acto, es lo que el Canadiense quiere discutir y, para ello, trae a colación un artículo del medievalista francés, Cajetan et l'existence, donde cree encontrar la razón por la que, según su parecer, tiene tantos problemas para probar la distinción.

\section{b) Distinción y relación entre esse y existencia}

Hasta el momento hemos dado cuenta de dos tesis principales de Lawrence Dewan, a saber, 1) la demostrabilidad de la distinción real a partir de la substancia causada, y 2) que debemos distinguir entre saber qué es el esse y su mera constatación como elemento distinto de la esencia, aunque nuestro conocimiento de él sea confuso y precario.

Aunque su reflexión adquiere en esta parte de su artículo una dinámica más bien circular, volviendo a estos temas principales, debemos considerar en este punto un tercer tema que se suma al conjunto, y que podríamos describir de la siguiente manera: Si Gilson declara firmemente que no es posible conocer la distinción real a partir de la substancia causada, y el objetivo es desentrañar los errores de su tomismo, hay que preguntarse dónde se fundamenta su escepticismo. La respuesta cree encontrarla en su artículo Cajetan et l'existence (1951), donde Gilson critica a Cayetano por identificar el esse tomista con la existencia real de la esencia. El Canadiense, en un primer momento, entiende que, en consecuencia, habría como un tercer elemento junto al esse; por un lado, estaría la esencia realmente existente y, por otro, su existencia real ${ }^{19}$. A su modo de entender, aquí estaría la razón por la que, supuestamente, algunos tomistas no quieren afirmar la demostrabilidad de la distinción. Tomemos pie de los textos de donde viene la confrontación.

The reason [por la que Dewan considera que Gilson no ve demostrable la distinción] is that he thinks of esse as something other than the actual existence of the essence (which actual existence he identifies with the essence). The true doctrine, I maintain, is that the actually existent essence is other than its own actual existence: and that can be demonstrated ${ }^{20}$.

Por su parte, Gilson sostiene que la existencia real de la esencia no es distinta ni separable de la misma esencia, por tanto, que esta existencia así llamada (real) no es el esse

${ }^{19}$ «Saint Thomas, que nous sachions, n'a jamais introduit de distinction ni de composition réelle entre l'essence et, quand elle existe, son 'être d'existence actuelle' L'être d'existence actuelle résulte de la composition d'une essence avec son acte d'être, il n'est pas un troisième élément que l'on puisse considérer à part». Gilson, É., «Cajetan et l'existence», Tijdschrift voor Philosophie, 15 (1953), pp. 267-286, en p. 272.

${ }^{20}$ Dewan, «Gilson» op. cit., p. 85. 
que se distingue de la esencia. Dewan entiende que el esse de Tomás es la existencia real de la esencia, que esta existencia de la esencia es el esse tomista; en cambio, piensa que Gilson, al negarse a ver en ésta el esse que compone con la esencia, está introduciendo como un tercer elemento.

Saint Thomas, as far as we know, has never introduced a distinction or a real composition between the essence, and, when it exists, its «being of actual existence». The being of actual existence results from the composition of an essence with its act of being, it is not a third element that one can consider separately ${ }^{21}$.

Con estas palabras, Dewan sitúa ahora el análisis del medievalista francés fuera del ámbito de un tercer elemento, pero dentro de otro contexto. Por su parte, parece evidente que Gilson está sugiriendo que el ser de existencia real es el resultado de la composición real de esencia y ser, y no el elemento que compone con ella. Comentando estas palabras, Dewan dice: «Certainly, it is not a third element. Esse is not other than the actual existence of the essence. The created essence has it as its proper act. The created essence, as long as it exists, is really distinct from its existence». Como se ve, rechaza la tesis de Gilson y afirma que, lo que Tomás llama esse, se identifica con la existencia real de la esencia, siendo este el elemento, por tanto, que compone con ella ${ }^{22}$. Dice aquí -y

${ }^{21}$ Gilson, «Cajetan», op. cit., p. 272.

${ }^{22}$ Que esta interpretación del esse como existencia proviene de Cayetano se prueba, directamente, por los mismos textos del cardenal: «En efecto, en el hombre hay que considerar la materia en que se recibe su forma, la forma misma, que es acto de tal materia, la esencia humana que no es ni la materia ni la forma, y el ser de la existencia actual por el que el hombre existe formalmente en la naturaleza de las cosas; de lo cual resulta que en tales sustancias se hallan dos composiciones, pertenecientes al género de sustancia, de las cuales la primera es la de materia y forma; la segunda, la de esencia y existencia, composición que se llama de ser y esencia». Cayetano, Tomás de Vio, O.P. Comentaria in De ente et Essentia, p. 139. Que Dewan se sitúa en la posición de Cayetano es algo que queda probado porque afirman lo mismo sobre el particular; además, el Canadiense no repara en elogios tanto a él como a Capreolo, a los que valida el título de «príncipe de los tomistas» (Dewan, «Gilson», op. cit., p. 85). Un trabajo que vincula directamente la identificación dewaniana del esse con la existencia con Cayetano: Muñoz, C., «Dificultades en torno a la interpretación cayetana del ens: esse essentiae y esse actualis existentiae», Themata. Revista de Filosofía, 49 (2014), pp. 235-244. Algunos estudios críticos con el esse existentiae de Cayetano: Gilson, «Cájetan», op. cit., pp. 267-286; Fabro, C. «L'obscurissement de l'esse thomiste», Revue Thomiste, 58 (1958), pp. 443-472; Hegyi, J., Die Bedeutyng des Seins bei den klassischen Kommentatoren des heiligen Thomas von Aquin. Capreolus - Silvester von Ferrara - Cajetan, Pullach, Verlag Berchmanskolleg. 1959; Duquesne, M., «Philosophie thomiste et lib(re recherche», Mélanges de science religieuse, 21 (1974), pp. 177-202; Llano, A. «Actualidad y efectividad», Estudios de Metafísica, 4 (1974), pp. 167-168; Echauri, R., «Esencia y existencia en Aristóteles», Anuario Filosófico, 8 (1975), pp. 117-129, en p. 128; más recientes: Orrego Sánchez, S., El ser en la escuela de Salamanca, Pamplona, Eunsa, 2004; Suárez, A., «Étienne Gilson y la distinción real de esencia y existencia en Santo Tomás de Aquino», Apuntes Filosóficos, 24/25 (2004), pp. 21-34, en p. 24; González Gatica, A., El pensamiento de Gilson sobre el «actus essendi» tomista, Roma, Pontificia Universitas Sanctae Crucis Facultas Philosophiae, 2010; García Cuadrado, J.Á., «Gilson y Báñez: luces y sombras de un encuentro tardío», Studia Gilsoniana, 5/4, (2016), pp. 579-618; del mismo autor y el mismo año «Domingo Báñez: entre la controversia 
esto es importante subrayarlo- «la tiene [la existencia real] como su propio acto». Es decir, la existencia real de la esencia es el acto de la esencia, el actus essendi.

L'esse, in quest'interpretazione [como existencia] è l'essere in atto dell'essenza, la sua realizzazione di fatto, il fatto del suo passaggio dalla possibilità alla realtà: l'essere è interpretato nella linea logico-formale secondo l'affermazione e la negazione semplice, come semplice «esistere» che ha contro di sè il non esistere, dove la potenza è ridotta a semplice «possibilità logica» che a sua volta nella sfera dell'esistenza viene relegata col non-essere. È per questo, con coerenza esemplare, che l'esistenza viene qualificata dal suarezismo per «contenuto nozionale» (il più perfetto!) grazie al quale tutto ciò ch'è si contrappone al nulla ed è in questo modo che viene interpretata l'attribuzione tomistica all'esse di essere la perfezione di tutte le perfezioni. L'esse in actu ha soppiantato l'esse [ut] actus, cioè l'esistenza ha eliminato l'esse ${ }^{23}$.

A continuación, el filósofo canadiense intenta comprender y explicar la tesis de Gilson de la cual acaba de tomar distancia. El esse parece significar para él un acto que pone a la esencia en el estado de existencia real, pero, este estado ni se distingue de la esencia ni es el esse. Para ello, cita otro texto donde el Francés intenta explicar su posición:

Thomas is not a disciple of Giles of Rome; he does not take esse for a thing; it is an act, but still it is for him an aliud about which it is literally true that the actual being [l'être actuel] is composed [out of it]. If, on the contrary, one has to do merely with existence, that is, the being of actual existence, one sees very well in what sense it is the actuality of the possible substance, but one no longer sees well how the actual substance is composed of it. It still remains permissable to ask oneself whether, for Cajetan, the Thomist esse is not reduced to the substance placed in the state of real existence [état d'existence réelle] by the efficacy of its cause? ${ }^{24}$

En contra de esto, los textos del Aquinate sugerirían, a su parecer, que la existencia como tal representa sin lugar a dudas lo que expresa perfectamente su noción de esse. ¿Cuál es la razón por la que Gilson se niega a aceptar la identificación, sin más, entre esse y existencia? Sin duda, sus esfuerzos parecen ir encaminados a mostrar que el esse

y el olvido», Azafea, 18 (2016), pp. 147-169; Ocampo, F., «El debate en torno al «Intellectus essentiae» y la «distinción real» entre la esencia y el ser en el De ente et essentia de Tomás de Aquino», Studia gilsoniana, 7/2 (2018), pp. 201-237; Marenghi, C., «El oscurecimiento del ser y su sustitución por la existencia», Studia Gilsoniana, 8/1 (2019), pp. 113-146.

${ }^{23}$ Fabro, Partecipazione, op. cit., p. 45.

${ }^{24}$ Gilson, «Cajetan», op. cit., p. 277. Del mismo parecer son todos los autores citados anteriormente, en cuyos trabajos rechazan que la existencia exprese el actus essendi. En concreto, sobre esta distinción entre acto y estado, dice Á. L. González: «El hecho de existir no es un principio metafísico, pues pertenece a un ente metafísicamente ya constituido, es decir, compuesto de acto de ser y esencia; en todo caso, es en el ente un efecto consiguiente a la posesión del esse. La existencia indica el ente en acto, la cosa que tiene una existencia actual, mientras el ser es aquello por lo que un ente es». González, Ser y, op. cit., p. 120. 
de Tomás de Aquino no se identifica con la esencia, como sucede en la Metafísica ${ }^{25}$ y en la escuela tomista posterior. La existencia es fruto de un acto, por lo que la existencia real es el estado fruto de ese acto (de esse), y no una perfección que goza el ente por la esencia ${ }^{26}$, como sucede en el paradigma aristotélico. Esta es la razón por la que parece que Gilson se niega a identificar el esse con la mera existencia real de la esencia, dado que el esse no es en sí la mera existencia, sino que ésta es la consecuencia del esse, concebido como acto que produce la existencia. La existencia, por tanto, sería el estado, entendido como efectualidad, producido por el esse, y no el acto del esse.

Mientras Heidegger equipara existencia con realidad o efectividad (Wirklichkeit) santo Tomás nunca utiliza existencia para indicar la esencia de Dios, porque entiende que su esencia consiste en ser, y utiliza el término esse. La existencia, como la entiende Heidegger, no es la naturaleza íntima de Dios, porque Dios no es una realidad o cosa. Dios es, para santo Tomás, actus purus, su esencia consiste en ser sin ser algo. No es acto puro como entiende Heidegger, como una realidad pura ya acabada y concluida, por lo que no admite ninguna posibilidad. Como se puede apreciar, no es posible identificar la existencia, o realidad efectiva, con el principio constitutivo del ente que hace real a la realidad. Puntualiza Echauri: la existencia señala para Heidegger el plano de la entidad, en tanto que para Santo Tomás el esse es lo que constituye a la entidad como entidad ${ }^{27}$.

La existencia, de suyo, no indica el actus essendi $i^{28}$; al contrario, indica el estado fáctico del ente que está ahí en el mundo, al modo del Dasein heideggeriano. La existencia de una casa, por ejemplo, nos dice que una casa existe en el sentido de que es eso, una casa existente que está ahí. Ahora bien, ¿cómo su mera existencia puede indicar su

${ }^{25}$ Me parece acertado el análisis de un estudio reciente que hemos citado antes sobre esta cuestión: «Más allá de su esencialismo de base, Aristóteles tiene el mérito de haber advertido que, en el ente, la esencia y la existencia no son lo mismo, porque el ser como tal sólo indica el hecho de existir. Y sin que siquiera lo haya sospechado, en su doctrina del acto y la potencia se encuentra el más profundo supuesto teórico de la distinción real entre esencia y ser que mencionará Boecio, vislumbrará Avicena y elaborará Tomás de Aquino». Marenghi, C., «La originalidad de la ontología tomista y su giro en torno al ser», Studia Gilsoniana, 7/1 (2018), pp. 33-69, en p. 38. Otro autor que apoya el origen remoto de la distinción en la teoría acto-potencia: Tweeten, D., «Really Distinguishing Essence from Esse», en G. Klima and A.W. Hall (eds.), Medieval Skepticism, and the Claim to Metaphysical Knowledge. Proceedings of the Society for Medieval Logic and Metaphysics, vol. 6, New Castle, Cambridge Scholars Publishing, 2011, pp. 79-129, en p. 114.

${ }^{26}$ Sobre la reducción del esse a la existencia, concretamente en la temprana escuela tomista, me remito a algunos trabajos citados antes (nota 22): Fabro, «L'obscurissement», op. cit., pp. 443472; Orrego Sánchez, El ser, op. cit., pp. 30-38; y añado aquí el más reciente: Marenghi, «El oscurecimiento», op. cit., p. 114.

${ }^{27}$ Gaya Berlanga, Vida y pensamiento, op. cit., p. 320.

${ }^{28}$ Además de Gilson, A. Maurer no tiene dudas sobre por qué el Aquinate no tradujo normalmente el esse como existentia. Cf. Maurer, A., «Revived Aristotelianism and Thomistic Philosophy», en V. Ferm, (ed.), A History of Philosophical Systems, New York, The Philosophical Library, 1950, pp. 197-211. Versión española, «La resurrección de Aristóteles y la filosofía tomista», A. Lema Hincapié (trad.), Praxis Filosófica, 16 (2003), p. 18. 
actualidad metafísica, su ser el acto supremo que produce esa existencia? Es difícil hacer derivar de la mera existencia la virtualidad propia del esse tomista, y, aunque admitiéramos la condición de Dewan de que sólo se trata de conocer que es un elemento distinto de la esencia, el problema que plantea Gilson parece irresoluble, porque, además, parece históricamente incuestionable: Aristóteles, que hacía de la esencia la actualidad suprema, no sólo no distinguió entre esencia y existencia sino que hacía derivar la existencia de la virtualidad (actualitas) de la propia esencia (esse in actu), algo palmariamente distinto a la filosofía del ser del Aquinate.

Nell'esperienza dell'ens ut ens c'è l'immediatezza di qualche natura in atto che ha cioè l'esse. La filosofia di ogni tendenza ha chiamato quest'esse esistenza, deformandolo, perchè esso è sperimentato propriamente come atto di una sostanza immediatamente data a questo: perchè la sostanza è ciò che è, perchè essa sola ha l'esse, perchè l'esse quindi s'impone alla coscienza come l'atto che pone e attua in se stessa la cosa ${ }^{29}$.

Una vez que el filósofo francés ha dejado claro que la mera existencia no traduce adecuadamente la virtualidad del esse $e^{30}$, vamos a adentrarnos en esta interesante distinción para discernir la relación entre esse y existencia. Hay algo que parece claro. Si, por una parte, la existencia entendida como factum no es el actus essendi, por otra, en cambio, algo tiene que ver con ella, puesto que el esse produce la existencia ${ }^{31}$, es un resultado -podríamos decir- de aquél. Se hace evidente aquí la necesidad de un fino análisis que se ocupe de esta relación.

Existence may mean either a state or an act. In the first sense, it means the state in which a thing is posited by the efficacy of an efficient or of a creative cause, and this is the meaning the word receives in practically all the Christian theologies outside Thomism, particularly those of Augustine, Boethius, Anselm, Scotus, and Suarez. In a second sense, existence (esse, to be) points out the interior act, included in the composition of substance, in virtue of which the essence is a «being», and this is the properly Thomistic meaning of the word ${ }^{32}$.

En una filosofía denominada esencialista, el esse es la existencia entendida como el estado resultante de la acción de una causa formal. En un segundo sentido, el de santo Tomás, existencia es el acto (acto de existencia), y no el estado derivado de aquél. Con esta distinción y relación que viene utilizando entre acto y estado, Gilson responde a esta dificultad. La clave hermenéutica parece clara: la existencia entendida como acto (acto de existencia) es la causa que produce la existencia de la cosa, la esencia existente. Como estado, la existencia es el resultado de lo que ese acto produce. El acto de existencia es la

${ }^{29}$ Fabro, Partecipazione, op. cit., p. 66.

${ }^{30}$ Filippi, S., «En torno a la Metafísica del Éxodo», Studia Gilsoniana, 4/2 (2015), pp. 99-115, en p. 117.

${ }^{31}$ Sobre la causalidad del esse se ocupará Dewan en el último apartado de su artículo, pero no concierne directamente a nuestro estudio.

${ }^{32}$ Gilson, Elements, op. cit., pp.130-131. 
condición de posibilidad de la existencia como estado de la esencia. Se trata, pues, de dos modos de concebir la noción de existencia, no de dos elementos distintos. Así parece sugerirlo el tomista español García López quien, haciéndose eco del debate en torno a la posibilidad o no de identificar ser y existir, afirma:

Algunos lo identifican [el existir] sin más con el ser, mientras que otros insisten en la irreductibilidad del ser al mero existir, o al mero hecho de existir, o de darse en la realidad. En este caso, lo correcto parece ser lo siguiente: el existir es como la cara externa o notoria del ser, pero no es realmente distinto de él; es como el efecto ${ }^{33}$ formal primario del ser (efecto formal quiere decir efecto intrínseco a la causa a la que se atribuye, y no separado, y ni siquiera distinto, de ella). En consecuencia, no hay ser sin existir, y tampoco existir sin ser. Si algo tiene ser, indudablemente existe, pero el ser no se reduce al mero existir, sino que es algo más, es la actualidad fundamental de cada cosa, y por ello, la perfección de todas las perfecciones o la perfección máxima ${ }^{34}$.

¿Un acto o un estado? Dewan se esfuerza por llegar a comprender qué quiere mostrar Gilson con esta distinción y en qué sentido puede aclararnos cómo podemos concebir el esse más allá de la existencia real de la esencia.

Anyway, the distinction between the «state» and the «act» is not all that clear. I take it that Gilson sees «state» as something that leaves the primary focus on the posited thing itself, whereas «act» would suggest something entering into the very composition of the thing or substance. I would only insist that, whether any other philosopher or theologian has ever recognized the distinction between the thing and its existence (what Gilson calls the «state» of the thing), Thomas sees the truth that there is a real distinction between the essence and its actual existence. This «state» is the actuality of being ${ }^{35}$.

Este texto tiene una importancia particular. Sea cual sea la comprensión exacta de lo que Gilson entiende con su distinción, Dewan tiene claro que, en los textos del Aquinate, la distinción se da entre la esencia y su existencia actual. La controversia podría surgir de su conclusión: «Este estado es la actualidad del ser». La contraposición con la tesis de Gilson es frontal. La actualitas del esse se corresponde con el estado de existencia de la esencia, tal y como encontramos en el tomismo de Cayetano, en parte de la escuela tomista posterior y en Suárez, algo que estaría muy alejado de la verdadera naturaleza del esse, según el Francés. A su modo de entender, es más correcto decir que el estado es la consecuencia de la actualidad del esse y no la actualidad misma del ser, de lo contrario, se empobrece la misma actualidad del esse al reducirla a la mera existencia. La existencia, de suyo, no comporta más perfección que la de su misma emergencia existencial (esse existentiae), pero, ¿a consecuencia de qué? ¿Qué actualitas ha hecho surgir esa existencia? Con criterio, Gilson distingue entre una metafísica de la esencia, que nos

\footnotetext{
${ }^{33}$ La cursiva es mía.

${ }^{34}$ García López, Lecciones, op. cit., p. 49.

${ }^{35}$ Dewan, «Gilson», op. cit., p. 91.
} 
responde a la pregunta quid est ens, de una metafísica del esse, que nos responde a la pregunta quo est ens.

Precisamente parece que, con el fin de no polemizar entre acto y estado, Dewan sugiere utilizar sencillamente actualitas como realidad más conforme con los textos de santo Tomás.

This verb «is» signifies that which occurs first to the intellect as having the status: absolute actuality [per modum actualitatis absolute]; for «is», said unqualifiedly, signifies being actually [esse actu], and so it signifies in the manner of a verb [per modum verbi]. But because the actuality [actualitas] which this verb «is» principally signifies is universally the actuality of every form or act, substantial or accidental, thus it is that when we wish to signify that any form or act is actually present in [actualiter inesse] some subject, we signify that by this word «is», unqualifiedly according to present time, in a qualified sense as regards the other times; and so, as a consequence, this verb «is» consignifies composition ${ }^{36}$.

A la luz de estos análisis, Dewan va perfilando su propia síntesis conclusiva: «My sense is that Thomas, in speaking of 'esse', is talking about what everyone talks about, but sees truths about it that others do not see». E inmediatamente, se confronta con Gilson citando y comentando un párrafo suyo:

How did Thomas come by this new notion? It is not a notion universally evident to all human minds. Far from it. The majority of philosophers will concede that it is a far cry from a possible thing to an actual thing. ... but if an actually existing being has been produced by its cause, why should one attribute to it an «existence» distinct from the fact that it exists? (...). No such disagreement would take place if the presence, in things themselves, of an act in virtue of which they can be called «beings» were a conclusion susceptible of demonstration ${ }^{37}$.

A la postura aparentemente más conciliadora del Canadiense que nos decía que hay una concepción más común de lo que se cree en el mundo intelectual acerca del esse, se contrapondría este radicalismo propio del tomismo gilsoniano, algo abrupto a juicio de ciertos filósofos, para los que esta noción original del Aquinate parecería inalcanzable. Pero lo que centra la atención de su análisis es lo que él interpreta como el escepticismo gilsoniano acerca de la demostrabilidad de la distinción, que es el estribillo común de esta disputa, y ante el cual él sigue proponiendo diferenciar entre el conocimiento del quid del esse y el esse como elemento distinguible, podríamos decir, de la esencia.

${ }^{36}$ Ibid., p. 92. A pie de página, cita Expositio libri Peryhermenias, comentando: «Thomas is explaining why the word is merely consignifies, and does not simply signify, composition. -Gilson, in Being and some Philosophers, p. 171-, uses actuality of both the esse and the form just in itself. Capreolus holds that one should reserve the word actualitas for esse. Defensiones 1.330a».

${ }^{37}$ Gilson, Element, op. cit., p. 131. 
Here, I would suggest that we might distinguish between knowledge of the act of being, in the mode of knowing called «that it is», and knowledge of it in the mode of «what it is». I think a text like the Quodl. 12 item on angelic esse is meant to answer the question of the «what?» regarding the act of being of caused things. Sometimes the people who speak of the "fact of existence» mean only «being» in the sense of the true. If they mean the very actuality of the substance, then they are talking about esse, and perceiving it, but not necessarily as to what it is ${ }^{38}$.

Es interesante de cara a la confrontación, reseñar los textos a los que nos reenvía Dewan para probar su tesis. Concretamente:

To see that one is confronted by a substance is to see a certain real unity of the manifold of sensible reality; and in seeing this unity, one is seeing an esse. Thus, as Aristotle says in De anima 2.1 (412b8-9): (...) for admitting that the terms unity [to hen] and being [to einai] are used in many senses, the paramount sense is that of actuality [he entelecheia]. And Thomas assures us (S.th 1, 11, 1, 56a45-47) (...) each thing, just as it guards its being [suum esse], so also it guards its unity [suam unitatem]. The substance or essence of the thing causes its unity, as we see in S. th. 1, 11, 4, obj. 3 and ad 3.

Según la particular interpretación de este texto por parte de Dewan, la percepción de la substancia nos acerca a la unidad real que reina entre todos los elementos que la componen. Lo que resulta extraño es que nos lleva a la Summa para hacernos ver que Tomás entiende que esta unidad, en acuerdo con Aristóteles, proviene de la esencia. Había citado antes el De anima queriendo indicar que en ambos autores coincidiría una común acepción del término ser como actualitas, olvidando -o ignorando- que, en estos dos filósofos, la palabra ser significan dos realidades metafísicas radicalmente distintas, pues, como es sabido, para Aristóteles, la actualitas, que manifiesta el ser y es garante de la unidad de la substancia, se corresponde con la essentia o forma; en cambio, para el Aquinate, actualitas es reservado, en un sentido supremo, originario y fundante al esse entendido como acto, precisamente del ente. Que Dewan pretenda decir a Tomás de Aquino con Aristóteles que la actualitas proviene en ambos de la substancia es una idea propia del tomismo cayetanista, que ha sido una de las tesis gilsonianas más acogidas por buena parte del tomismo contemporáneo.

Llegamos así al punto donde realmente comienza la comprensión de toda la metafísica, el esse, a la luz bien del ens o bien del Esse puro. Gilson dice: «It is one and the same thing to conceive of God as pure Esse and to conceive things, so far as they are, as including in their metaphysical structure a participated image of the pure Act of Being». Y Dewan comenta:

Now, this is true in the case of Thomas. However, I do not think that one comes to the metaphysical conception of God as the subsistent act of being until one sees that the hierarchy of efficient causes cannot go to infinity. I say this because it seems to me that

\footnotetext{
${ }^{38}$ Dewan, «Gilson», op. cit., p. 93. Cita Quodlib. 12, 5, 1 (12) y manda a S. th., I, 3, 4 ad 2.
} 
the distinction between a thing and its act of being is seen as soon as one sees the contribution of the efficient cause as cause. There must be, in the effect, both a nature of its own and a participation in what is proper to the nature of the cause as cause. These cannot be identical. But that there is some one being whose nature is esse is seen in seeing that there is a first cause, first by nature ${ }^{39}$. [En nota al pie añade:] See the previously mentioned Gregorianum paper. -I do ask myself, however, if we could not use a Fourth Way approach- (i.e. S. th. 1, 2, 3: The Fourth Way), and see a composition of esse and essence because of the more and the less in beings: cf. SCG 2, 15. Still, this merely raises the question: how different, one from another, are the Five Ways? ${ }^{40}$.

Este párrafo de carácter claramente sintético y conclusivo causa cierta perplejidad. Dewan dice que esto-lo dicho por Gilson- es cierto en el caso de Tomás (como si el objeto de su discusión con él no fuera sobre el modo más acertado de expresar el pensamiento de Tomás). Sin embargo, a continuación, introduce el siguiente argumento -de difícil continuidad hermenéutica-: «no se puede llegar a la noción de Dios como Acto puro de Ser si no es a través de la jerarquía de causas eficientes que no pueden ir al infinito». De este razonamiento cabe hacer, al menos, dos consideraciones importantes. Una es que, expresándose así, el que parece alejarse del camino metafísico andado por Tomás para sentar las bases de su comprensión del esse sería el propio Dewan. Otra es que, argüir que a Dios, como Ser Subsistente, se llega por la jerarquía de causalidad eficiente, además de no ser el camino de Tomás ni en la Summa ni en el Contra gentiles, habría que decir que es la vía que trazó Aristóteles, un filósofo que de ningún modo concibió que la Causa Primera de los entes sea el Ser mismo Subsistente (ipsum Esse), al contrario, como prueba su aporía undécima, declaró la imposibilidad de tal cosa ${ }^{41}$. Él insiste en que la distinción se ve a partir de la causalidad eficiente como responsable de la existencia de los entes. Pero, como ya hemos mostrado, Aristóteles concebía la substancia como causada pero, en cambio, no vio la distinción. Quedaría por añadir, o quizá, más bien, objetar, algo sobre su mención al hecho de la participación de los entes en el Ser de Dios. ¿No presupone la noción de participación la de Acto puro de Ser, siendo una petición de principio hacer derivar de aquí la distinción? Si hay distinción porque el ente es una participación del Esse puro, ¿no sería Éste, como insiste Gilson, la condición de posibilidad de que podamos concebir la substancia creada como una participación de Él (del Esse puro) y, por tanto, a la luz de este Esse puro, concebir la distinción?

${ }^{39}$ Dewan, «Gilson», op. cit., p. 94.

${ }^{40}$ Nota 71 de la misma página 94.

${ }^{41}$ Idea que habría que matizar diciendo que, desde un marco metafísico donde el orden superior de actualidad es el de la esencia, la doctrina del ipsum Esse se hace aporética, no porque sea impensable, como algunos dicen, sino porque la noción de Esse puro de Tomás de Aquino no surge en ese marco (del esse ut essentiae) sino en otro (el del esse ut actus). 


\section{Valoración sistemática de los argumentos}

Me ha parecido oportuno dedicar un apartado a la consideración sintética de las ideas principales que se han venido desarrollando, primero sobre la distinción real y, después, sobre la noción de existencia como concepto adecuado para expresar el esse.

\section{a) La distinción real desde la substancia}

El estudio atento de la obra de Gilson no deja lugar a dudas sobre el hecho de que el argumento de la composición real de esencia y ser tomista fue uno de los más defendidos por él a lo largo de su carrera, razón por la cual esta acusación de Dewan resulta altamente cuestionable. Por ello, con el fin de aclarar un aspecto importante de la confrontación, hay que diferenciar entre probar un argumento y demostrarlo. Ambas cosas son distintas, pero no se oponen, de tal manera que se puede probar un principio, pero no demostrarlo, como sucede con el famoso principio de contradicción aristotélico. Como explicaba el Estagirita, la imposibilidad de demostrar un principio se debe a su condición de originario o fundante del resto de principios, nunca a un escepticismo o negacionismo. Lo mismo podemos aplicar respecto de nuestro problema, puesto que podemos probar la distinción real pero no demostrarla. Gilson probó que Tomás de Aquino defendió y probó mediante sus escritos la distinción real de esencia y ser, pero no encuentra una demostración filosófica, que es lo que pretende Dewan.

Entonces ¿por qué un autor como Gilson, firme defensor de este principio capital del tomismo, se negó a defender una demostración filosófica de la distinción? La respuesta ilustrará la tesis que hemos tratado de exponer. Primeramente, el motivo más importante es que quiso desmarcar la argumentación tomista de la explicación aviceniana -sobre la que Tomás pudo haberse apoyado sobre todo al principio de su carreray ello por dos razones: una, para evitar la doctrina de la accidentalidad; otra, para no hacer derivar su fundamento de un principio ajeno a su propia filosofía, en este caso, su original comprensión del esse, algo extraño a Avicena. En segundo lugar, puesto que la clave hermenéutica que permite la comprensión tomista de la distinción se basa, según Gilson, en el esse, el particular estatuto epistemológico de éste desemboca en la indemostrabilidad filosófica. Es decir, puesto que el núcleo de la distinción recae sobre el conocimiento del esse, y, a su vez, el conocimiento del esse es posible pero indemostrable, se sigue de ahí que la distinción también es indemostrable.

Esto nos lleva a recordar que el tema del conocimiento del actus essendi ha sido polémico. Desde la posición suareciana, que negaba la distinción real porque veía en el esse una esencia al interpretar el aliud por otra cosa, hasta la teoría intuicionista de Maritain. En el primer caso, Gilson argumenta que el esse es un acto y no una esencia (cosa) por lo cual de él no podemos formar concepto ni tener un conocimiento habitual u ordinario del mismo. 
El error fundamental [de los tomistas], dice Suárez, es que incurren en una petición de principio. Cuando les pregunta: «¿Cómo podéis saber qué es la existencia?», ellos responden planteando la distinción de esencia y existencia como condición del tal conocimiento. Pero, ¿cómo distinguir la esencia de la existencia, si todavía no sabemos qué es la existencia? Este último argumento es probablemente el más iluminador de todos, por lo que respecta a la propia posición de Suárez ${ }^{42}$.

Lo que le gustaría saber es quid existentia sit: qué es la existencia, como si la existencia fuera un qué. Habiendo identificado el ser con su esencia, no podía jamás hallar en él un es que, si es, no fuera una esencia ni una cosa. En el segundo caso, respecto a la explicación de Mariatain, el rechazo de Gilson se basa en que esta teoría es ajena al pensamiento del Aquinate, por lo que él personalmente no se siente cómodo asumiendo un argumento que no se encuentra en sus textos.

En resumidas cuentas, uno tiene la impresión de que Dewan no distingue entre el conocimiento de un principio y su demostrabilidad filosófica. Del esse no podemos tener un conocimiento evidente y sintético-abstractivo por su misma naturaleza.

Aunque son justificables, no debemos permitir que tales fórmulas nos lleven a pensar, equivocadamente, que el «ser» (esse) es una cosa. El ser (esse) es lo que hace que una esencia sea «un ser», y, puesto que la esencia necesita recibirlo para ser, aún teniendo su propio acto de existencia, sigue siendo distinta de él $1^{43}$.

Es necesaria una indagación metafísica, como sucede en otros casos, pero ello no es razón suficiente para descartar su peculiar estatuto epistemológico. Al contrario, lo que estas páginas, a través de las cuales Dewan ha deconstruido el discurso de Gilson para tratar de probar la distinción por la sola substancia, no han conducido sino a la corroboración del acierto del Francés. Su conclusión dista mucho de lo que entiende su opositor.

Esta tesis fundamental implica consecuencias de largo alcance. Porque, si bien Tomás de Aquino no la propuso como un medio para distinguir a los seres finitos de Dios, una vez propuesta, resolvió inmediatamente toda la cuestión. Si hay distinción entre esencia y existencia en todos y cada uno de los seres, entonces todo ser es distinto de Dios en virtud de la composición que le hace ser «un ser» $»^{44}$.

En este texto se ilustra con claridad que la distinción real sí se puede probar (que no demostrar) pero no desde los seres finitos sino desde Dios, que es de donde el Aquinate construye su filosofía del ser. Al expresarse de este modo, Gilson trata de corregir la postura acerca de la distinción que defendió al principio de su carrera, pues así creía avanzar en fidelidad al pensamiento de santo Tomás. Por eso, cuando se le hizo ver que realmente el Aquinate no fundamentaba la distinción a partir de la substancia sino de

\footnotetext{
${ }^{42}$ Gilson, É., El ser y los filósofos, Pamplona, Eunsa, 2005, p. 205.

${ }^{43}$ Ibid., p. 223.

${ }^{44}$ Ibid., p. 224.
} 
Dios, se decantó por la corrección sin ambages. Se lo exigía su deseo de atenerse minuciosamente a lo que él creía como el verdadero espíritu del tomismo. Parece quedar en evidencia, pues, que Dewan necesita recurrir al conocimiento del esse para justificar la distinción, y sus maniobras para tratar de evitarlo recurriendo a la ambigüedad en torno a la profundidad de este conocimiento no han hecho más que poner de relieve el embarazo explicativo al que se llega por ese camino.

De otra parte, me parece oportuno dedicar un comentario específico a uno de los textos del Canadiense que hemos utilizado durante la confrontación porque, a través de su análisis, se evidencia precisamente dicha dificultad ${ }^{45}$.

La argumentación dewaniana parte de una premisa muy discutible: que para probar la distinción no es necesario empezar distinguiendo el ser de la esencia. Valoremos a continuación si es posible descartar de entrada la distinción del esse respecto de la esencia, recurriendo sencillamente al hecho de una causalidad indeterminada. Dewan afirma: «Ambos términos [esse y esencia] parecen referirse a los principios o elementos de un ser». La utilización del verbo parecer aquí sugeriría indeterminación argumentativa. Por otra parte, ¿cuál es la razón por la que estamos autorizados a concebir dos elementos, y además distintos? Aristóteles sólo vio uno, la esencia existente (esse in actu). Para el Estagirita, el ente es la esencia existente ${ }^{46}$. Nada más. Si de aquí pareciera derivarse una distinción entre dos elementos distintos, no hay razón alguna para pensar que él no los viera, teniendo en cuenta que a este filósofo debemos el análisis más profundo del ente.

Y concluye: «Es solo cuando la cosa se ve como causada y, por lo tanto, depende de una esencia más elevada y más noble, que uno debe concebir una distinción, dentro de la cosa causada, entre su esencia y su acto de ser». Este argumento sugiere que la causalidad substancial («una esencia más elevada») -en clara alusión a la causalidad substancial aristotélica- nos conduce de suyo a la distinción. Pero caigamos en la cuenta del juego lingüístico y metafísico de Dewan. Habla de una causa más noble de la substancia para fundamentarla, pero sólo nos la describe como una «esencia más elevada y más noble», sin especificar más acerca de ella. Es claro. No puede hacerlo, porque sabe que si habla de ella especificando su verdadera naturaleza caería en el error de una petición de principio, puesto que estaría dando por supuesto que esa causa es puro Ser, lo que le conduciría a admitir lo que pretende negarle a Gilson. Por lo demás, aparte de esta pequeña sutilidad, el argumento no prueba lo que afirma. Del hecho de que la substancia sea causada no se sigue la distinción, a no ser que, por el previo conocimiento de la naturaleza de esa causa, sepamos que la substancia, por la particular relación ontológica con esa causa, lo sugiriera. Pero este no es el caso, puesto que se habla de una causalidad donde lo único que se especifica de su naturaleza es la esencia. En una palabra: estamos en la causalidad substancial aristotélica ${ }^{47}$, de la cual no se deriva que podamos conocer

${ }^{45}$ Vid. supra, nota 6, p. 3.

${ }^{46}$ «L'ens in actu è per Aristotele l'essenza realizzata». Fabro, Partecipazione, op. cit., p. 174.

${ }^{47}$ Suárez, A., «Étienne Gilson y la distinción real de esencia y existencia en Santo Tomás de Aquino», Apuntes Filosóficos, 24/25 (2004), pp. 21-34, en p. 24. 
distinción alguna en la substancia. Este razonamiento conduce a un callejón sin salida, porque, como por la causa esencial no puede hacer derivar en la distinción de ambos elementos, queda, como Aristóteles, en esta causalidad formal. La única manera de traspasar esta causalidad es sencillamente a través del conocimiento de la naturaleza del esse. Si hay una causalidad que puede hacernos ver la distinción es la eficiente transcendental (la del esse), y esta se funda en la noción de Dios como Acto puro de Esse ${ }^{48}$.

En resumen: el conocimiento de la substancia fruto de una causalidad substancial sin referencia alguna a la actualidad originaria del esse tanto puro como participado, de ningún modo conduce a la distinción de esencia y ser. La prueba evidente -como se ha venido afirmando- es que el filósofo que descubrió la substancialidad y su causalidad en el Acto puro, no sólo no distinguió en la substancia la esencia de la existencia, sino que, además, hacía derivar la existencialidad de la misma esencia ${ }^{49}$, fiel a su metafísica en la que el acto supremo es el de la esencia. La existencia es aquí un resultado de la perfección de la esencia ${ }^{50}$. No distinguió a una de la otra, pero, de haberlo hecho, la habría juzgado, como Avicena, como un accidente de ella ${ }^{51}$. En cualquier caso, no se pueden utilizar los conceptos de esencia y causalidad sin especificar en qué orden o paradigma metafísico los enmarca porque de eso depende que podamos o no probar la distinción. Lo que queda claro es que, en tal caso, introduciéndonos en ese otro orden transcendental, la condición de posibilidad de la distinción ya no es ni la mera substancia ni su mera causalidad substancial sino el esse.

En la doctrina de Avicena, el necesario por sí mismo causa los necesarios por otro; en la doctrina de Tomás de Aquino, el Ser por sí mismo causa seres por vía de causa. Esta es la razón de que, partiendo del análisis de Avicena del ser finito, pueda concluirse con la distinción de Avicena entre esencia y existencia, y éste es un método del que Tomás ha hecho buen uso; pero no puede concluirse con la distinción tomista de esencia y existencia porque, bajo su forma propiamente tomista, la distinción presupone la noción de esse concebido en las substancias concretas como el más alto principio intrínseco de su mismo ser ${ }^{52}$.

${ }^{48}$ Un estudio crítico del tomismo gilsoniano que advierte también de esta necesaria referencia al esse para traspasar la causalidad formal aristotélica, Paván, C., «Gilson lector de Santo Tomás: apuntes metafísicos». Apuntes Filosóficos, 5 (1994), pp. 37-46, en p. 46.

${ }^{49}$ Echauri, «Esencia», op. cit., p. 128.

${ }^{50}$ «El esse puede estar en la esencia, y nosotros veremos cuán estrecho se halla ligado a ella, pero no es nunca algo de la esencia». Gilson, É., El ser y la esencia, Buenos Aires, Emecé, 1962² p. 97.

${ }^{51}$ «Forma ed atto, forma e perfezione si corrispondono e coincidono in Aristotele senza residui: per questo non poteva l'esse avere in Aristotele un'emergenza propria, ma esso declina nell'essenza, è trasferito nell'entità dei reali: non ha senso perciò nella metafisica aristotelica una trattazione a parte dell'esse, perchè essa ignora sia il significato contingentistico di existentia dell'avicennismo latino, sia quello di atto primo e originario di San Tommaso». Fabro, Partecipazione, op. cit., p. 162.

${ }^{52}$ Gilson, El ser y los filósofos, op. cit., p. 163. La cursiva es mía. 
En este caso, necesariamente se está operando con un conocimiento del esse, sin el cual no se puede traspasar el orden esencial, como de hecho nadie antes de Tomás hizo en sentido propio.

Sorprendentemente, a continuación, Dewan nos dice: «Es verdad que, si uno no reconoce el esse como un objetivo de atención metafísica desde el principio, nunca propondrá la doctrina tomista». Esta afirmación debilita fuertemente el sentido de su argumentación y viene a concluir en la aporía que acabamos de mostrar. Es más, habría que matizar que no sólo no propone la doctrina tomista, sino que tampoco prueba lo que dice. Pero la sutilidad de los términos que emplea debe atraer nuestra atención. Empezaba diciendo que no es necesario ver la distinción del esse desde el inicio. Ahora dice que debe ser reconocido «como un objetivo de atención metafísica desde el principio». Aquí habría que preguntar, por un lado, si esto no contradice lo primero y, por otro, qué quiere decir exactamente «reconocer el esse como un objetivo de atención metafísica». Se deja ver en este vago modo de expresarse una indeterminación forzada cuyo objetivo es dejar al lector en una ambigüedad intencionada. ¿Cómo se puede reconocer el esse desde el principio, dentro y fuera de la doctrina tomista si no es acudiendo a la fuente de donde brota la entraña de su misma esencia? Si el conocimiento que podemos tener de él es confuso, como aseguraba él mismo, esto nos hace tener que recurrir a la causalidad substancial, pero viendo que aquí estamos en Aristóteles y no en Tomás, el argumento nos acorrala y nos pone necesariamente de cara a la naturaleza del esse como fundamento de la distinción.

«Sin embargo, eso no significa que uno deba conocer completamente la naturaleza del esse desde el principio». En esta conclusión vemos cómo ha quedado fuera la ambigüedad de «reconocer el esse como objetivo de atención metafísica» y afirma con más claridad la vuelta a su tesis inicial de que «no hay necesidad de conocer la natu-raleza del esse desde el principio». Hay que reparar, sin embargo, en la introducción del adverbio modal completamente, empleado aquí con un significado que le tiene que servir para su argumentación y por el que, por tanto, nos tenemos que interrogar. La tesis sostiene que no tenemos que conocer completamente la naturaleza del esse desde el principio. De entrada, conduce al absurdo, porque, una cosa o se conoce o no se conoce, y es evidente que una cuestión de naturaleza cualitativa-como en este caso saber o no saber cuál es la naturaleza de un principio-, no tiene sentido plantearla desde el punto de vista cuantitativo -si su conocimiento es más o menos completo, puesto que se trata de saber o no saber sobre él. Incluso planteado desde el punto de vista cualitativo, la cuestión conduce al absurdo, porque si hay algún tipo de conocimiento del esse, ¿qué podríamos conocer desde el punto de vista cualitativo sobre él de cara a afrontar qué papel juega ese tipo de conocimiento en el conocimiento de la distinción? Es una cuestión cualitativa. Si lo que conocemos del esse es su substancialidad, entonces hay que decir, desde el punto de vista de Tomás de Aquino, que en este caso no conocemos el esse. Cualquier cosa que podamos conocer con más o menos profundidad de él, si no es su condición de acto y acto supremo, no nos dice realmente qué es. Y en tal caso, eso que podemos saber de él no nos dice qué es, por lo que, de cara a la cuestión de la distinción, no podemos distinguir 
de la esencia un elemento del que, lo que podemos saber es todo menos que es su acto (del ente), por tanto, distinto de la esencia.

\section{b) Esse y existencia}

A lo largo de la exposición de la argumentación mediante la cual Dewan ha querido fundamentar su crítica a la posición gilsoniana en torno a la cuestión de la posibilidad o no de identificar esse y existencia, ha quedado en evidencia que el Canadiense desconoce de algún modo que esta cuestión ha sido discutida desde que la filosofía del ser de Tomás de Aquino vio la luz del día. En cambio, recientes estudios muestran que ya en la Escuela de Salamanca, los principales representantes de esta importante época del tomismo emplearon indistintamente esse y existencia para referirse al actus essendi. Pero la cuestión que nosotros hemos tratado de discutir aquí no ha sido exactamente esta. Lo que aquí hemos estudiado es si el esse de Tomás de Aquino se corresponde con el estado de existencia real de la esencia, tal y como defiende Lawrence Dewan. Nuestra indagación nos ha permitido conocer la posición de Gilson al respecto, y la necesidad de enfocar el problema desde una perspectiva más amplia, como hacen otros autores, excepto Fabro, el cual se muestra completamente intransigente respecto a la utilización de existencia para referirse al esse. En cambio, hay autores, desde la mencionada Escuela de Salamanca, que utilizan existencia considerada no como el mero estado de existencia real de la esencia, como hace Dewan (quizá influenciado por el suarecismo y el cayetanismo), sino como acto, pero no entendiendo esta actualitas reducida al mero estado existencial de la esencia, a lo que se opone la mayor parte del pensamiento tomista. Como se ha tratado de mostrar, si bien el acto de ser está vinculado esencialmente con el estado existencial del ente, sin embargo, reducirlo a la mera existencia es, como se dijo, un empobrecimiento que de ninguna manera se corresponde con la doctrina del esse de santo Tomás.

Hay quien sitúa la clave hermenéutica del asunto en la distinción entre el orden lógico y el orden real. La consideración metafísica del esse entendido como raíz de toda actualidad correspondería al orden real y no al lógico, propio de las proposiciones, al cual estaría vinculada la constatación de la existencia fáctica.

La existencia tomada en este sentido [lógico] por tanto, es de un contenido ontológico paupérrimo; todo lo que pudiera haber en ella de positividad y perfección le vendría de la esencia. Por lo tanto, se puede decir con razón que siempre que se ha entendido la existencia como el modo de la «efectividad» se está muy lejos de la comprensión tomista del ser como acto y raíz de todas las perfecciones ${ }^{53}$.

Aunque el ser veritativo (de las proposiciones) alcance el ser real no hay manera de ver, desde aquí, cómo alcanzar el esse como acto de todos los actos, raíz de toda perfección. Tal y como hemos querido mostrar partiendo de las afirmaciones de Gilson, el

\footnotetext{
${ }^{53}$ Orrego Sánchez, El ser, op. cit., p. 32.
} 
problema está en querer pasar de la mera substancia existente (esse in actu) al porqué o razón metafísica de la actualidad existente en esa substancia, de hecho, existente. Nosotros formulábamos esta cuestión con la pregunta ¿qué actualidad produce la existencia real de la esencia?, puesto que el recurso dewaniano a la causalidad ya vimos que era irresolublemente aporético, por cuanto que, por una parte, sin una explícita mención al tipo de causalidad (si es la eficiente tomista o la substancial aristotélica) nada podemos dilucidar, por lo que habríamos de decantarnos por una de ellas y, por otra, la misma causalidad eficiente no puede ser respuesta adecuada porque Dios, única causa eficiente, causa la existencia de la substancia a través del esse como lo primero en la constitución real del ente. El recurso a la causalidad eficiente propio de Dewan no responde al entramado metafísico porque se sale del marco analítico del ente. Dios es causa eficiente del ente como único Agente que interviene en su producción, sin embargo, dentro del marco analítico comprensivo propio del estudio metafísico, la causa eficiente, Dios, opera a través de la creación ${ }^{54}$, que es la producción del esse a partir del cual la substancia recibe, como fuente originaria, toda su actualidad, tanto existencial como formal. Dios crea el esse, y este esse es la actualidad que, participada del Esse puro, confiere a la substancia su ser, formal y existencial. La pregunta que Gilson hace a quienes interpretan el tomismo desde la esencia es, ¿cómo puede accederse a la naturaleza del esse así entendido por la sola substancia creada? Dewan había dicho que no se trata de la substancia como substancia sino ésta como creada por la eficiencia de una causa. Ya se argumentó que esta explicación, desde el momento en que se acaba apoyando necesariamente en la comprensión tomista del esse, no consigue deconstruir la explicación gilsoniana sino todo lo contrario, admitirla. Por tanto, desde la substancia existente sólo podemos constatar, con Aristóteles, que es (fácticamente) tal cosa (su esencia), pero nunca su estatus de acto primero y fundante de toda la realidad.

Esta orientación del debate tiene su origen en el estudio de lo que se conoce como tomismo analítico, que ha sido estudiado en el ámbito del tomismo español por el filósofo Alejandro Llano, quien ha dedicado varios trabajos a esta cuestión, dentro de la cual está nuestro problema, considerado desde este marco comprensivo.

Y ya con el objeto de resumir el status questionis de nuestro debate, cabe señalar que existen dos posturas, equivalentes en lo substancial, pero confrontadas con aquella que hemos visto defendida por Dewan. Por un lado, se encuentran, a la cabeza: Cornelio Fabro y Gilson; y junto a ellos: Millán-Puelles, García López, Á. L. González, Echauri, Livi, Raeymaecker; y más en la actualidad: Llano, Canals, Forment, Redpath, Possenti, Tarasiewicz, Herrera, Filippi, C. Muñoz, quienes consideran que el traspaso hacia existencia

${ }^{54}$ «La producción trascendental del esse por vía de creación no anula, sino que exige, como se ha visto, la mediación del recipiente propio del esse. Esta exigencia surge de la tensión dialéctica del ipsum esse como plexo de todas las perfecciones». Ferraro, C., «La interpretación del esse en el tomismo intensivo de Cornelio Fabro», Espíritu, 153 (2017), pp. 11-70, en p. 59. 
ha sido la causa de la desviación formalista ${ }^{55}$. Por otro, aquellos donde ha anidado un tomismo más formalista cercana a las posiciones cayetanistas, al menos respecto de la identificación entre esse y existencia: Dewan, Quinn; y en la actualidad, algunos autores que han seguido la línea dewaniana: Brock, Irízar y Torrijos.

\section{Conclusiones}

Después de esta presentación de las principales tesis que conforman la confrontación entre Lawrende Dewan y Étienne Gilson en torno a la demostrabilidad del esse y su distinción con la esencia, estas son las conclusiones a las que hemos ido llegando, y que ahora resumimos e modo de conclusiones.

Para ver en el esse un principio completamente distinto de la esencia y no derivado de ella es absolutamente necesario advertir su verdadera naturaleza metafísica, esto es, el ser acto supremo en el orden entitativo. Sólo así se puede concluir en una distinción auténticamente real respecto de la esencia y no un accidente de ella.

En la metafísica de Tomás de Aquino, el orden formal explica la causalidad y la substancialidad de los entes. Al orden transcendental o existencial sólo es posible acceder a través del conocimiento de la naturaleza real del esse, algo a lo que el Aquinate llegó progresivamente, pero de un nuevo paradigma ontológico, al que pudo acceder a través de la revelación de Ex 3, 14 y de la noción de creación.

Puesto que el esse es un acto y no una esencia (cosa), su conocimiento no puede derivar de un proceso de abstracción o conceptualización, como sucede con los objetos comunes de conocimiento intelectual, porque pretender esto sería convertirlo en lo que no es, una cosa (esencia) objeto de conocimiento, cuando en realidad es un acto, de lo cual no podemos tener conocimiento conceptual. En este sentido decimos que el esse no puede ser demostrado, sino que a él debemos acceder por otros caminos especulativos ${ }^{56}$, desmarcándose de teorías ajenas a los textos de Tomás. No parece convincente acceder a él, como pretende Dewan, aplicando la doctrina del conocimiento del alma, la cual, como forma, es acto primero y supremo en el orden substancial, pero en el orden del esse, como sucede con toda forma, es acto segundo.

Por tanto, es lo inapresable del esse por su naturaleza lo que impide una demostrabilidad así entendida de la distinción real de esencia y esse desde la substancia causada.

De igual manera y con relación a esto, el recurso a la causalidad tampoco logra traspasar la distancia cualitativa entre estos dos órdenes metafísicos. Nada autoriza desde la filosofía del ser de santo Tomás de Aquino a deducir el orden del ser del orden de la

${ }^{55}$ Romera Oñate, L., «Verso un pensiero dell'essere: dialogo tra Heidegger e Fabro», Acta Philosophica, 1/1 (1992), pp. 101-110, en p. 102.

${ }^{56}$ Gilson cree que el camino adecuado es el juicio existencial. 
substancia, tampoco en el orden causal. La causalidad desde la substancia explica la substancialidad del ente, no que en el ente podamos distinguir un acto que le hace existir. Por su parte, la causalidad eficiente, que es la del esse, presupone la noción de esse como elemento cualitativo determinante que nos hace situarnos más allá del orden de la substancia. Cuando invocamos, entonces, esta causalidad, ya no estamos en la substancia causada sino en la substancia causada a través del esse.

El recurso a la consideración del aspecto dual del esse como acto (ser) y como factum (existencia) no condujo a Dewan sino a una esencialización del esse, de la mano de Capreolo, Cayetano y Suárez. La existencia, si por un lado evidencia connaturalmente el esse, sin embargo, no puede agotar ni explicar por su solo aspecto fáctico la riqueza del esse. Parece más acertada la explicación de Gilson de que la existencia real de la esencia no es todavía sino un estado de actualidad de la esencia y por la esencia, pero no aún el actus essendi, que es el esse concebido como la energía original causal que produce esta existencia (acto). Esta dialéctica entre ser-existencia como acto-estado parece, insisto, una explicación más detallada y específica que la mera identificación calletanista del actus essendi con la existencia real de la esencia.

Por tanto, creo, finalmente, que las páginas de Gilson and the Actus Essendi consiguen el resultado contrario a su objetivo, ya que, a mi parecer, ayudan a reforzar las ideas del tomismo gilsoniano y ponen en evidencia las que conforman el tomismo dewaniano.

Manuel Alejandro Serra Pérez manuel.serra@um.es

Fecha de recepción: 20/05/2019

Fecha de aceptación: 02/07/2019 